\title{
Simulation of sound propagation over porous barriers of arbitrary shapes
}

\author{
Guoyi Ke and Z. C. Zheng ${ }^{\text {a) }}$ \\ Department of Aerospace Engineering, University of Kansas, Lawrence, Kansas 66045
}

(Received 13 December 2013; revised 16 November 2014; accepted 25 November 2014)

\begin{abstract}
A time-domain solver using an immersed boundary method is investigated for simulating sound propagation over porous and rigid barriers of arbitrary shapes. In this study, acoustic propagation in the air from an impulse source over the ground is considered as a model problem. The linearized Euler equations are solved for sound propagation in the air and the Zwikker-Kosten equations for propagation in barriers as well as in the ground. In comparison to the analytical solutions, the numerical scheme is validated for the cases of a single rigid barrier with different shapes and for two rigid triangular barriers. Sound propagations around barriers with different porous materials are then simulated and discussed. The results show that the simulation is able to capture the sound propagation behaviors accurately around both rigid and porous barriers.
\end{abstract}

(C) 2015 Acoustical Society of America. [http://dx.doi.org/10.1121/1.4904553]

[DKW]

Pages: 303-309

\section{INTRODUCTION}

The primary advantage of time-domain simulation is its ability to accommodate a wide variety of physical effects on acoustic propagation, including boundary-medium effects, scattering by turbulence, refraction by shear and temperature gradients, and diffraction over terrain. For example, many authors have already used finite-difference time-domain (FDTD) algorithms to simulate acoustic propagation. ${ }^{1-9}$ Under realistic environmental conditions, trees, bushes, buildings, and hills can influence sound propagation. Sound propagation around objects with complicated geometry is a challenging problem, and additional computational techniques are needed. The immersed boundary method employed in this paper provides an effective technique to tackle this problem.

Numerical techniques have been developed to study sound propagation over terrain, such as the ray-tracing method. However, the ability and accuracy to include irregular geometries and realistic wind profiles is limited. We have previously developed immersed-boundary (IB) methods ${ }^{10-12}$ that can be used to accommodate complex geometries with irregular shapes, multiple objects with different media, and even moving objects. These methods have been successfully implemented to compute the flow induced pressure fluctuations. ${ }^{11,12}$ In this paper, we extend the algorithm to calculate acoustic propagation using the linearized Euler equations. The acoustic barriers are considered as porous media, and the acoustic propagation is governed by the Zwikker-Kosten (Z-K) equations. ${ }^{13}$ There have been many studies on diffraction over barriers in the literature, such as those in Refs. 14-23. They are all theoretical, analytical, and numerical solutions from the boundary element method and therefore have restrictions in shape and distribution of the barriers.

\footnotetext{
a) Author to whom correspondence should be addressed. Electronic mail: zzheng@ku.edu
}

The simulation presented in this paper has the advantage of no restrictions in all these aspects.

\section{MODEL DESCRIPTION}

The geometry and coordinate system of a model problem of sound propagation over a sound barrier can be illustrated as in Fig. 1. At the top boundary, a perfectly matched layer (PML, Refs. 24-26) is used to allow an ideally impedance-matched propagation into the top boundary. The detailed implementation of the PML for this study can be found in Ref. 6. Combining the linearized Euler sound propagation equations in the air ${ }^{1}$ with those equations in a porous medium in the form of the $\mathrm{Z}-\mathrm{K}$ equations, ${ }^{13}$ we have

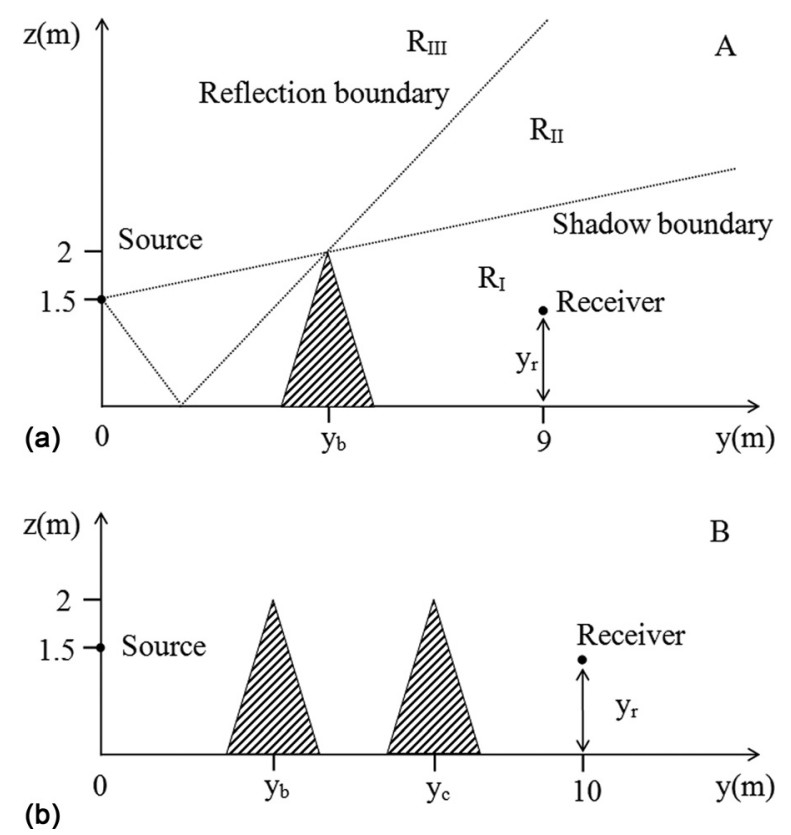

FIG. 1. Geometry A with a single triangular barrier, and Geometry B with two triangular barriers that refer to cases 1 and 2, respectively. 


$$
\begin{aligned}
& \frac{\partial \mathbf{u}}{\partial t}+\left(\mathbf{u}_{a v} \cdot \nabla\right) \mathbf{u}+(\mathbf{u} \cdot \nabla) \mathbf{u}_{a v}=-\alpha_{a v} \nabla p-\alpha \nabla p_{a v}+f_{\mathbf{u}}, \\
& \frac{\partial p}{\partial t}+\left(\mathbf{u}_{a v} \cdot \nabla\right) p+(\mathbf{u} \cdot \nabla) p_{a v}=-\gamma p \nabla p-\gamma p_{a v}(\nabla \cdot \mathbf{u})+f_{p},
\end{aligned}
$$

where $f_{\mathbf{u}}$ and $f_{p}$ are fictitious body forces to enforce the velocity and pressure to accommodate the governing equations in air and the Z-K equations ${ }^{13}$ inside a rigid or porous object and are given by

$$
f_{\mathbf{u}}= \begin{cases}0 & \text { Outside the porous medium } \\ \alpha_{a v} \nabla p+\alpha_{a v} \nabla p+\left(\mathbf{u}_{a v} \cdot \nabla\right) \mathbf{u}+(\mathbf{u} \cdot \nabla) \mathbf{u}_{a v}-\frac{\Omega}{c_{s}} \alpha_{a v}(\nabla p+\sigma \mathbf{u}) & \text { Inside the porous medium }\end{cases}
$$

and

$$
f_{p}= \begin{cases}0 & \text { Outside the porous medium } \\ \gamma p \nabla p+\gamma p_{a v}(\nabla \cdot \mathbf{u})+\left(\mathbf{u}_{a v} \cdot \nabla\right) p+(\mathbf{u} \cdot \nabla) p_{a v}-\frac{\gamma p_{a v}}{\Omega}(\nabla \cdot \mathbf{u}) & \text { Inside the porous medium. }\end{cases}
$$

In the preceding equations, $\mathbf{u}_{a v}, p_{a v}$, and $\alpha_{a v}$ are the time averaged velocity, pressure, and specific volume, respectively; and $\mathbf{u}, p$, and $\alpha$ are their acoustic fluctuations, with

$$
\alpha=-\frac{p}{\gamma p_{a v} \rho_{a v}},
$$

where $\gamma$ is the specific-heat ratio. The intent is to group terms to present wave propagations in air as the usual simulation, and wave propagation in porous media as the modified simulation with the fictitious body forces.

With the model equation system represented by Eqs. (1) and (2), arbitrary geometries of objects, rigid or porous, can be simulated without a complicated grid mesh arrangement. This is enabled by introducing the fictitious body forces, $f_{\mathbf{u}}$ and $f_{p}$, in the equations following the IB methods. ${ }^{10}$ In the IB methods, the equations are typically discretized on a Cartesian grid. The methods generally do not require that the geometry of the structure conform in any way to this Cartesian grid, which is the grid mesh used in the simulation. The advantage of the immersed-boundary method is that there are no interface boundary conditions needed. The interface conditions are embedded in the arrangement in Eqs. (1)-(4). Near the interface, the grid points inside or on the boundary of the porous media are calculated through the Z$\mathrm{K}$ equations, while the grid points outside the porous media are calculated through the equations in air. This operation is implemented automatically in the computational program via the expressions of the fictitious forces in Eqs. (3) and (4). The detailed implementation of the IB methods can be found in Refs. 10-12.

In addition, sound propagation in both the fluid medium and the porous medium can be calculated using a single, combined scheme. In this study, we present three different geometries of barriers such as screen, triangular barrier, and rectangular barrier, to test our scheme, and double barriers are also studied. The fluid medium is the air, and the porous media include the ground and the porous barrier. As the ground boundary is aligned with the Cartesian grid mesh, the barrier is the primary motivation for introducing the IB concept into the simulation.

\section{NUMERICAL SCHEMES}

Equations (1) and (2) are solved using the finite differencing schemes. For spatial derivatives, a forward scheme is employed for the velocity, and a backward scheme is employed for the pressure,

$$
\begin{aligned}
& \left(\frac{\partial p}{\partial y}\right)_{i, j} \approx \delta p=\left(\frac{p_{i, j}-p_{i-1, j}}{\Delta y}\right), \\
& \left(\frac{\partial \mathbf{u}}{\partial y}\right)_{i, j} \approx \delta \mathbf{u}=\left(\frac{\mathbf{u}_{i+1, j}-\mathbf{u}_{i, j}}{\Delta y}\right) .
\end{aligned}
$$

A first-order, forward-time scheme is applied for the time derivatives,

$$
\begin{aligned}
& \frac{\partial p}{\partial t} \approx \frac{p^{n+1}-p^{n}}{\Delta t}=Q\left(\delta \mathbf{u}^{n}, \delta p^{n}, \mathbf{u}^{n}, p^{n}\right), \\
& \frac{\partial \mathbf{u}}{\partial t} \approx \frac{\mathbf{u}^{n+1}-\mathbf{u}^{n}}{\Delta t}=P\left(\delta \mathbf{u}^{n}, \delta p^{n+1}, \mathbf{u}^{n}, p^{n}\right),
\end{aligned}
$$

where $P$ and $Q$ are the linear operators, and $\delta$ represents the first-order spatial differencing defined in Eqs. (6) and (7).

The stability of the numerical scheme has been studied in Ref. 6. For objects with a large flow resistivity, a very small time step is required. In that case, the scheme in Eq. (9) for the porous medium is modified to an implicit scheme for velocity to ensure an acceptable time step,

$$
\frac{\partial \mathbf{u}}{\partial t} \approx \frac{\mathbf{u}^{n+1}-\mathbf{u}^{n}}{\Delta t}=P\left(\delta \mathbf{u}^{n}, \delta p^{n+1}, \mathbf{u}^{n+1}, p^{n}\right) .
$$

It should be noted that there are high-order schemes in the literature. ${ }^{7,9,27}$ Schemes similar to the current one, 
accurate to first-order in time and second-order in space, have been used in the literature. ${ }^{1,6}$ They usually require more grid points per wavelength and smaller time steps than higher order schemes. In the present study, the computing power available is capable of meeting the resolution requirement. In addition, the interface between the air and the porous medium requires a high resolution to reduce the interface errors.

\section{EXPLANATION OF CASES}

In this section, we discuss the cases that are tested. Also we specify the geometries of single and double barriers, different locations of receivers, and different materials of barriers.

First, to compare with analytical solutions, several cases of sound propagation over a rigid, single barrier with different shapes are considered. A large flow resistivity value of $20000 \mathrm{kPa} \mathrm{s} \mathrm{m}^{-2}$ is selected in the $\mathrm{Z}-\mathrm{K}$ equation simulation to represent a rigid barrier. Figure 1 shows the geometries of barriers A (single triangular barrier) and B (double triangular barrier). In Fig. 1 and following text, the notation $(y, z)$ denotes horizontal position $\mathrm{y}$ in meters and height $\mathrm{z}$ in meters. In all the cases, the source is located at $(0,1.5)$. We compare the numerical results with the analytical solutions, obtained from the geometrical theory of diffraction, ${ }^{14,15}$ at different locations of the receiver. In geometry A, there is a triangular barrier between the source at $(0,1.5)$ and the receiver at $\left(9, \mathrm{y}_{\mathrm{r}}\right)$. The shadow boundary and reflection boundary divide the receiver space into three zones as $R_{I}, R_{I I}$, and $\mathrm{R}_{\mathrm{III}}$. In the shadow zone $\mathrm{R}_{\mathrm{I}}$, only diffraction waves and their reflections can reach there. In the zone $\mathrm{R}_{\mathrm{II}}$, additional direct waves from the source can also arrive there. In the zone $\mathrm{R}_{\mathrm{III}}$, diffraction waves, direct waves, as well as the first source reflection from the ground, can all arrive there. Among all the cases investigated, we selected results from the two cases listed in Table I as the representative cases to report here. In case 1 (geometry A) in Table I, two different locations of receivers are specified: $(9,1)$ in $R_{I}$ and $(9,3)$ in $R_{I I}$. Only the results in the zones $R_{I}$ and $R_{I I}$ are presented here, as particularly $R_{I}$ is the most sensitive to simulation errors with the barrier. More discussions about sound diffraction by single and double edges can be found in Refs. 17 and 19, and the analytical solutions for case 1 with a rigid ground are also provided in these two papers.

Sound wave propagation over two rigid barriers is also calculated in our simulation, as illustrated with Geometry B in Fig. 1 and represented by case 2 in Table I. We present results for a receiver at $(10,1)$ in the zone $R_{I}$ and a receiver $(10,3)$ in the zone $\mathrm{R}_{\mathrm{II}}$. The geometry of this case has two separated edges, so that we need to consider one more diffraction path for the theoretical analysis when the receiver is above the barrier. The analytical solution for this case can be obtained from Ref. 19.

We have also tested other geometries for the barrier, such as rectangular and screen barriers. We found that the results obtained for the triangular geometries are typical and similar trends can be found in other geometries. We therefore focus on the triangular geometries in this paper to be concise.

Finally, different materials for the barriers are considered. The geometry of the triangular barrier in case 1 is simulated for different materials. For that, the flow resistivity $\sigma$ of the barrier materials is set to 2,20 , and $200 \mathrm{kPa} \mathrm{s} \mathrm{m}^{-2}$. It is noted that according to Ref. 1, for typical absorbing ground materials, the flow resistivity approximately ranges from 10 to $100 \mathrm{kPa} \mathrm{s} \mathrm{m}^{-2}$.

\section{SIMULATION RESULTS AND DISCUSSION}

In this study, we use a Gaussian impulse source as the initial condition, with an expression of

$$
p(r, t=0)=\exp \left(-40 r^{2}\right), \quad \mathbf{u}(r, t=0)=0,
$$

where $r$ is the distance from the source position, with $p$ in pascals and $r$ in meters.

For the air, we have the values of $p_{a v}=100 \mathrm{kPa}$, $\gamma=1.4$, the speed of sound of air $c=340 \mathrm{~m} / \mathrm{s}$. When considering the barriers and the ground as porous media, we specify the porosity $\Omega=0.3$, and the porous medium structure factor $c_{s}=3$, following those specified in Refs. 1 and 6 .

For the single barrier case 1 with the rigid ground, the size of the simulation domain is $16 \mathrm{~m} \times 12 \mathrm{~m}$. The size of the grid of both $\Delta y$ and $\Delta z$ is $0.01 \mathrm{~m}$, except near the barrier at $\mathrm{z}$ $\in[1,5]$ and $y \in[2,7]$ where the size of grid is $0.0025 \mathrm{~m}$. The time step $\Delta t$ is $2.5 \times 10^{-6} \mathrm{~s}$, and the overall simulation time is $40 \mathrm{~ms}$. For the porous triangular barrier, we use the same grid arrangement and the time step, only adjusting the flow resistivity $\sigma$ inside the barrier. When the flow resistivity is large at the value of $20000 \mathrm{kPa} \mathrm{s} \mathrm{m}^{-2}$ (while the other porous medium parameters remain the same), the semi-implicit scheme expressed in Eq. (10) is used. The small grid size warrants a sufficient number of grid points within a wavelength, approximately 30 points for the highest frequency of interest at $1000 \mathrm{~Hz}$. The small time step not only satisfies the stability condition of the scheme ${ }^{6}$ and the Nyquist rule for the high frequency requirement, but also gives a low CFL number that is needed to avoid generating spurious waves near the interface between the air and the porous medium.

We increase the simulation domain for the double triangular-barrier case to $20 \mathrm{~m} \times 12 \mathrm{~m}$. The grid size is almost the same as in case 1 except at $y \in[2,8]$ where the size of the grid is $0.0025 \mathrm{~m}$. The time step is $2.5 \times 10^{-6} \mathrm{~s}$, and the overall simulation time is $45 \mathrm{~ms}$.

TABLE I. Specification for the two cases illustrated in Fig. 1.

\begin{tabular}{lcccc}
\hline \hline Case & Vertex points in each geometry $(\mathrm{m})$ & $\mathrm{y}_{\mathrm{b}}(\mathrm{m})$ & $\mathrm{y}_{\mathrm{c}}(\mathrm{m})$ & Receiver $\mathrm{y}_{\mathrm{r}}(\mathrm{m})$ \\
\hline 1 & $\mathrm{~A}(3.5,0),(4,2),(4.5,0)$ & 4 & None & $1,1.5,2,3$ \\
2 & $\mathrm{~B}[(4.5,0),(5,2),(5.5,0)],[(7.5,0),(8,2),(8.5,0)]$ & 5 & 8 & $1,1.5,2,3$ \\
\hline \hline
\end{tabular}


To illustrate how sound waves propagate over a single rigid barrier, we look at case 1 as an example, shown in Fig. 2. At time zero, the computation starts with a Gaussian impulse source. Figure 2(a) shows that the waves diffract around the barrier. Part of the waves below the height of triangular barrier reflects back from the rigid barrier, and the top part of the waves goes around the barrier. The reflected waves propagate in the opposite direction, while the top part of the waves produces another ground reflection when it touches the ground in Fig. 2(b). In the contours in Fig. 2(b), there are two groups of signals, represented by the wave fronts, lines 1-4.

Each group contains two lines: The first one (lines 1 and 2) represents the original sound wave where line 2 is the first reflection from the ground. The second group (lines 3 and 4) is due to the existence of the barrier that bends the first group and causes another reflection from the ground. The other cases not presented here with a single rigid barrier have similar behaviors.

To determine the relative sound pressure levels and compare with the analytical solutions, we performed a freefield computation for sound propagation of the same impulse source to obtain sound pressure $p_{\text {free }}$ and then use the following formula to obtain the relative sound pressure level:
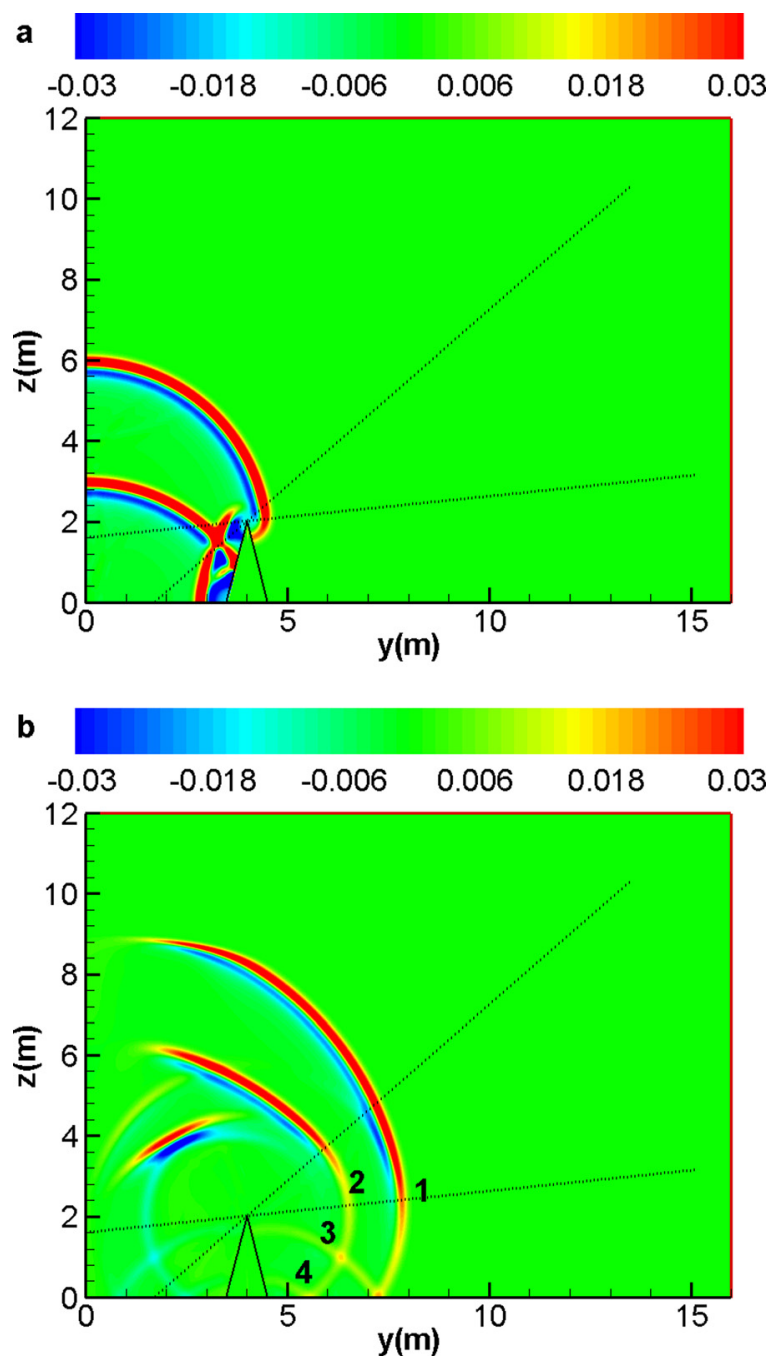

FIG. 2. (Color online) Pressure contours at different times for case 1, (a) $13 \mathrm{~ms}$ and (b) $23 \mathrm{~ms}$.

$$
\Delta L=10 \lg \left(p / p_{\text {free }}\right)^{2} .
$$

The relative sound pressure spectral results for case 1 and comparisons with the analytical solutions are presented in Fig. 3. The numerical results agree very well with the analytical solutions in all the receiver locations for a wide frequency range from $0 \mathrm{~Hz}$ to $1 \mathrm{kHz}$. Although not shown here, all the other single rigid barrier cases also show very good agreement in comparison to the analytical solutions in all the receiver locations in the $0-1 \mathrm{kHz}$ frequency range.

For the double-rigid-barrier case, two groups of signals in Fig. 2(b) will diffract again and become four groups of signals due to another rigid barrier. At time $26 \mathrm{~ms}$ in Fig. 4(a), the waves diffract around the second rigid barrier, and at the same time, a part of the waves reflects back from this barrier. At time $42 \mathrm{~ms}$ in Fig. 4(b), double diffractions around rigid barriers generate four group of the signals represented as lines $1-8$. The pattern of the following waves is very complicated because of interactions between waves and two rigid barriers. The results of relative pressure spectrum for the double rigid-barrier case are presented in Fig. 5 in comparison to the analytical solutions. A good agreement between the simulation and the analytical results is again achieved, although the overall agreement is more accurate in the single rigid barrier cases. A possible reason is that the original group of signals diffracts twice and becomes four groups of signals because of the two edges in the doublebarrier case, which may decrease the accuracy of the numerical simulation.
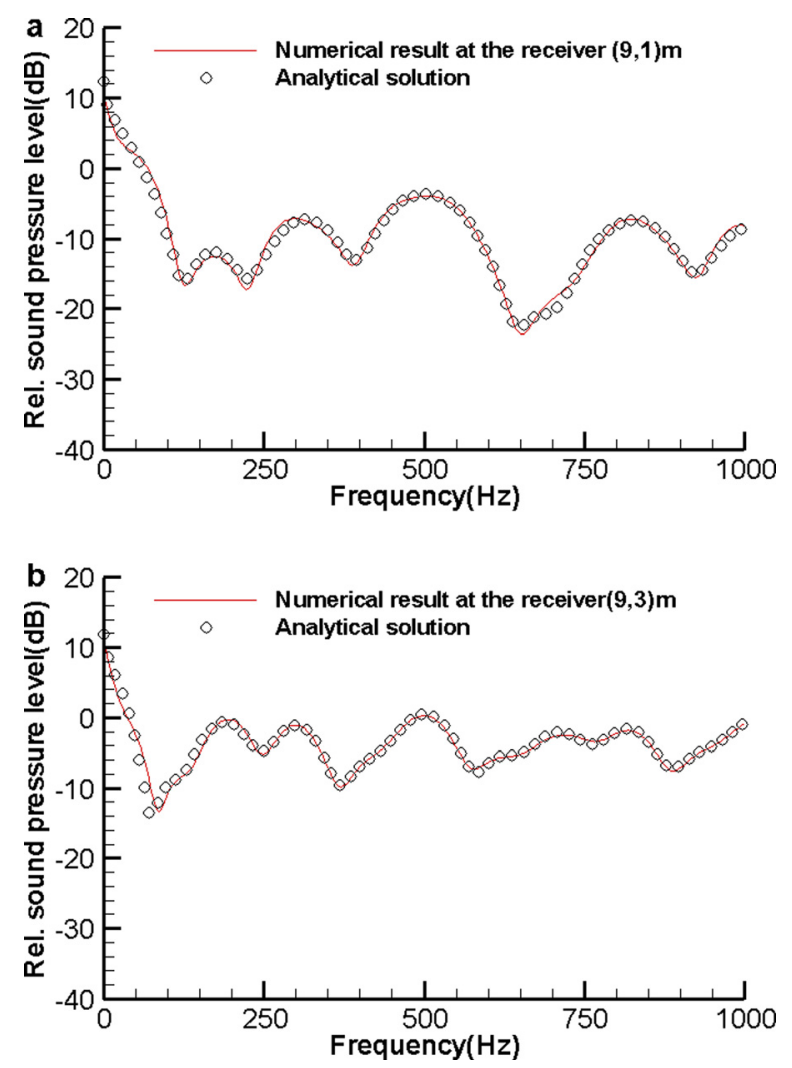

FIG. 3. (Color online) Comparison of numerical results with analytical solutions for case 1 , (a) $\mathrm{y}_{\mathrm{r}}=1 \mathrm{~m}$, (b) $\mathrm{y}_{\mathrm{r}}=3 \mathrm{~m}$. 
a
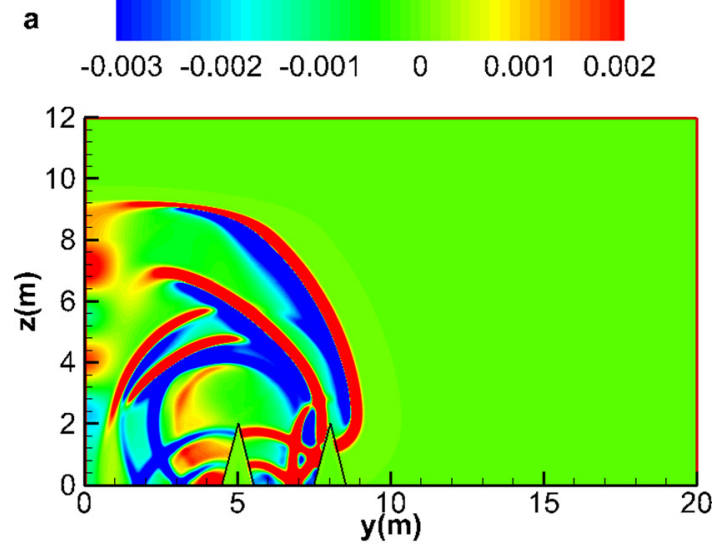

b
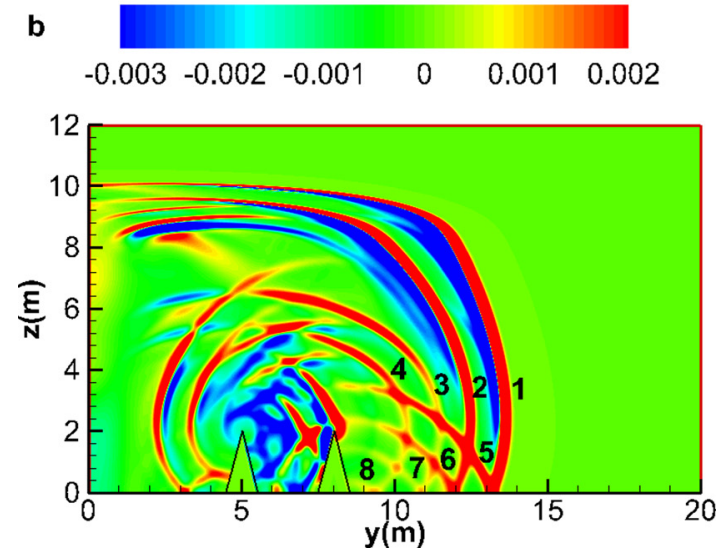

FIG. 4. (Color online) Pressure contours at different times for case 2, (a) $26 \mathrm{~ms}$ and (b) $42 \mathrm{~ms}$.
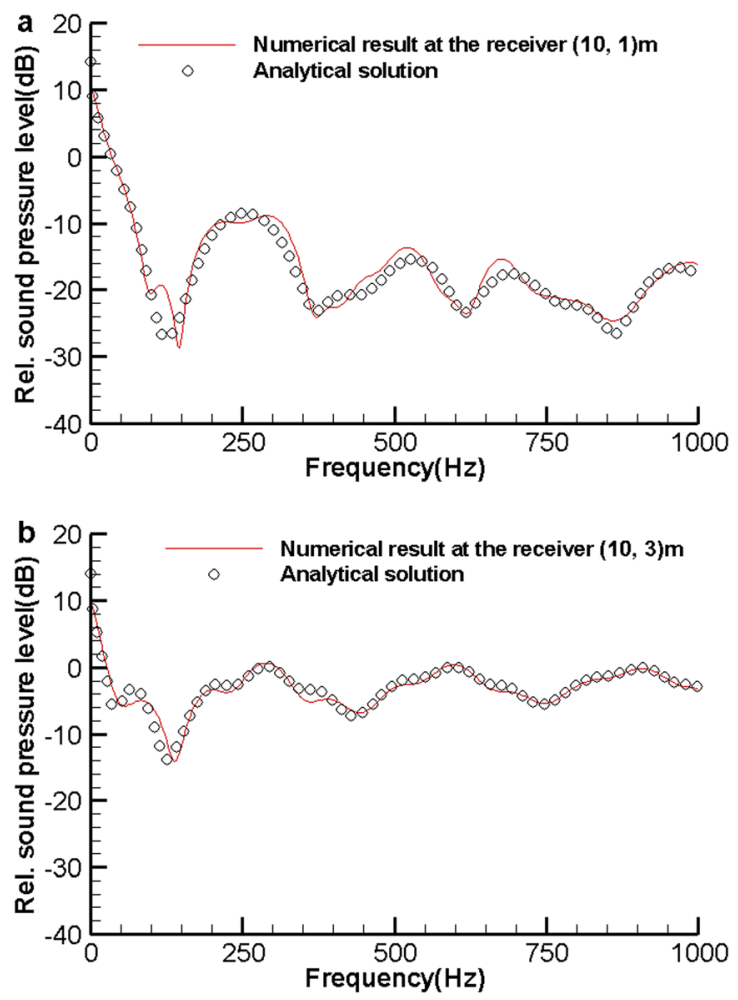

FIG. 5. (Color online) Comparison of numerical results with analytical solutions for case 2, (a) $\mathrm{y}_{\mathrm{r}}=1 \mathrm{~m}$, (b) $\mathrm{y}_{\mathrm{r}}=3 \mathrm{~m}$.
The flow resistivity of the porous barriers significantly influences the acoustic field. Barriers with a high flow resistivity, such as that of $\sigma=200 \mathrm{kPa} \mathrm{s} \mathrm{m}^{-2}$, behave just like rigid barriers. All sound waves below the height of the barrier are almost reflected. On the contrary, for low flow resistivity such as $2 \mathrm{kPa} \mathrm{s} \mathrm{m}^{-2}$, the sound waves can penetrate the barrier as illustrated in Fig. 6(a). It is obvious that the waves transform in the porous barrier due to the decreased speed of sound, which is $c / \sqrt{c_{s}}=196 \mathrm{~m} / \mathrm{s}$, much smaller than that in the air. ${ }^{28}$ As a result, the original group of signals in Fig. 6(b) is not separated by the barrier from the group of waves propagating through the barrier, contrary to the rigid barrier case shown in Fig. 2(b) where the two groups differ clearly. Corresponding to the relative pressure spectrum results in Fig. 7, the levels obtained for the flow resistivity of $2 \mathrm{kPa} \mathrm{s} \mathrm{m}^{-2}$ are much higher than those of the other flow resistivity cases because the barrier with low flow resistivity is much easier for sound waves to go through. The results for other shapes of barriers with different flow resistivities show similar trends.

Figure 8 provides the pressure variation with time for the triangular barriers with different flow resistivities. At the same receiver location, the wave amplitude corresponding to
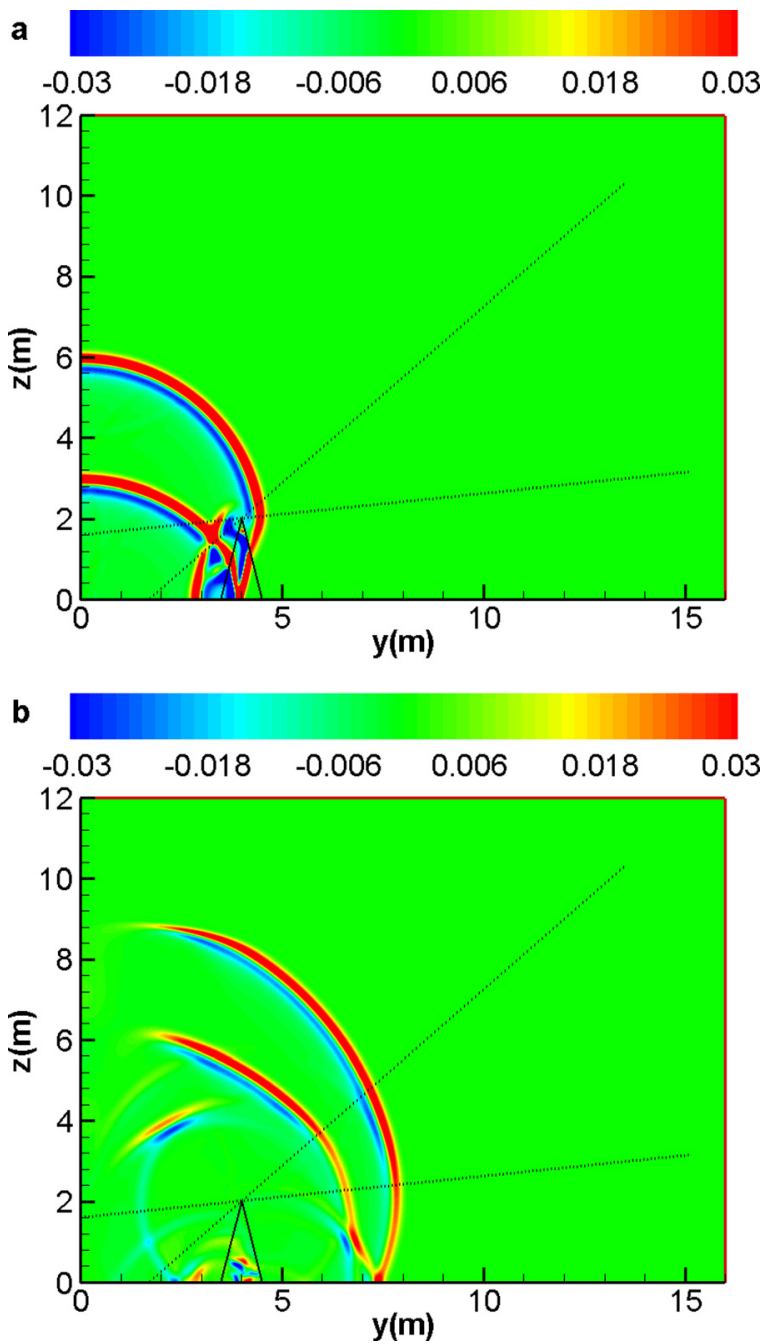

FIG. 6. (Color online) Pressure contours at different times for a triangular barrier with flow resistivity $2 \mathrm{kPa} \mathrm{s} \mathrm{m}^{-2}$, (a) $13 \mathrm{~ms}$ and (b) $23 \mathrm{~ms}$. 

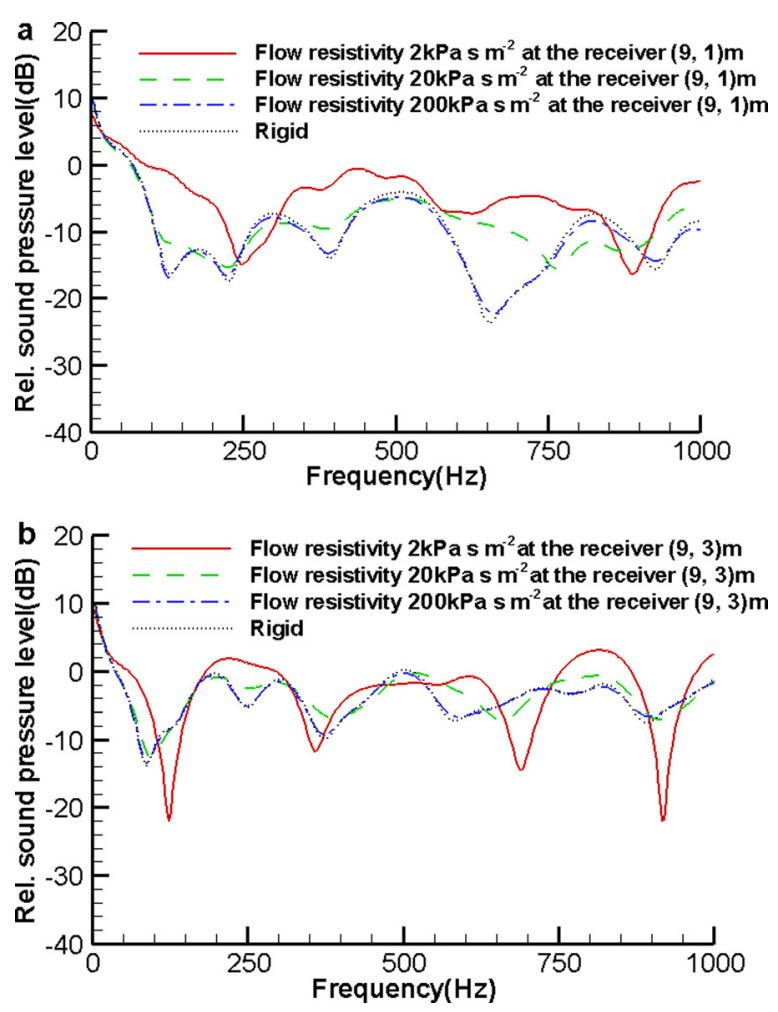

FIG. 7. (Color online) Numerical results for a triangular barrier with different flow resistivities (a) $\mathrm{y}_{\mathrm{r}}=1 \mathrm{~m}$ and (b) $\mathrm{y}_{\mathrm{r}}=3 \mathrm{~m}$.

the low flow resistivity case is generally greater than that of high flow resistivity, which means more sound energy propagates to the receiver and sound waves are easier to get through the barrier with a low flow resistivity. At the receiver $(9,1)$ in Fig. 8 , the amplitude of the first peak of wave increases as the flow resistivity decreases. However, at the receiver $(9,3)$, not much variation of amplitude is observed in the first peak of wave although there is a significant difference at the second peak. Comparing the second peak of wave at these two different receiver locations, at the receiver $(9,3)$ the second peak occurs much later than at the receiver

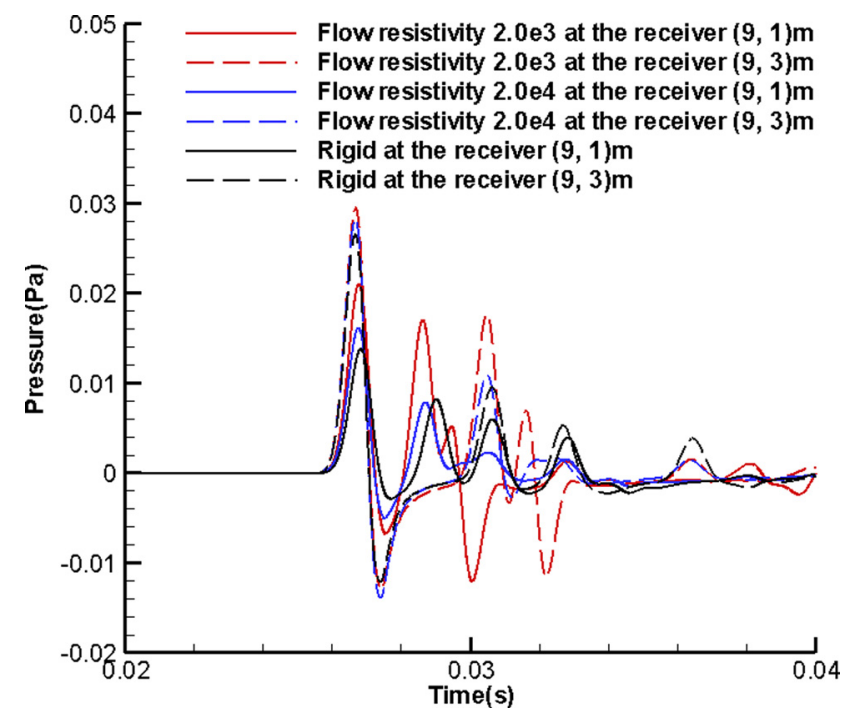

FIG. 8. (Color online) Waveform in time series at different receivers for a triangular barrier with different flow resistivities.
$(9,1)$. Therefore the late arrival of the second wave peak at the receiver $(9,3)$ shows that the phase of wave in the zone $\mathrm{R}_{\mathrm{II}}$ is distorted due to wave diffraction.

\section{CONCLUSION}

An accurate and effective acoustic propagation solver has been developed that combines the governing equations for acoustic propagation in air and in porous media. It is accomplished by making use of the immersed-boundary method so that the propagation around barrier objects with arbitrary shapes can be simulated. Behaviors of acoustic propagation over barriers with different shapes, different materials, and different arrangement were studied and discussed, and the numerical results have been validated by comparing with the analytical solutions. Comparisons with the analytical solutions show that the simulation results are particularly accurate in the shadow zones where diffracted waves interact with the reflected waves from the ground as well as with transmitted waves in the case of a porous barrier. In the shadow zone, the lower flow resistivity barrier gives higher relative sound pressure level because of the highly penetrable porous material of the barrier. Waveforms also show that wave diffraction results in phase distortion in the shadow zone.

\section{ACKNOWLEDGMENT}

This research is partly supported by U.S. Army under a cooperative Agreement No. W911NF-11-2-0087.

${ }^{1}$ E. M. Salomons, R. Blumrich, and D. Heimann, "Eulerian time-domain model for sound propagation over a finite-impedance ground surface. comparison with frequency-domain models," Acta Acust. Acust. 88, 483-492 (2002).

${ }^{2}$ T. Van Renterghem and D. Botteldooren, "Numerical simulation of the effect of trees on downwind noise barrier performance," Acta Acust. Acust. 89, 764-778 (2003).

${ }^{3}$ D. K. Wilson, V. E. Ostashev, and S. L. Collier, "Time-domain equations for sound propagation in rigid-frame porous media," J. Acoust. Soc. Am. 116, 1889-1892 (2004).

${ }^{4}$ V. E. Ostashev, D. K. Wilson, L. Liu, D. F. Aldridge, N. P. Symons, and D. H. Marlin, "Equations for finite-difference, time-domain simulation of sound propagation in moving inhomogeneous media and numerical implementation," J. Acoust. Soc. Am. 117, 503-517 (2005).

${ }^{5}$ D. K. Wilson, V. E. Ostashev, S. L. Collier, N. P. Symons, D. F. Aldridge, and D. H. Marlin, "Time-domain calculations of sound interactions with outdoor ground surfaces," Appl. Acoust. 68, 173-200 (2007).

${ }^{6}$ Z. C. Zheng and W. Li, "Numerical stabilities and boundary conditions in time-domain Eulerian simulations of acoustic wave propagations with and without background flow," Appl. Math. Comput. 202, 146-161 (2008).

${ }^{7}$ M. Hornikx, R. Waxler, and J. Forssèn, "The extended Fourier pseudospectral time-domain method for atmospheric sound propagation," J. Acoust. Soc. Am. 128, 1632-1646 (2010).

${ }^{8}$ G. Guillaume, J. Picaut, G. Dutilleux, and B. Gauvreau, "Time-domain impedance formulation for transmission line matrix modeling of outdoor sound propagation,” J. Sound Vib. 330, 6467-6481 (2011).

${ }^{9}$ D. Dragna, B. Cotté, P. Blanc-Benon, and F. Poisson, "Time-domain simulations of outdoor sound propagation with suitable impedance boundary conditions," AIAA J. 49, 1420-1428 (2011).

${ }^{10} \mathrm{~N}$. Zhang and Z. C. Zheng, "An improved direct-forcing immersed-boundary method for finite difference applications," J. Comput. Phys. 221, 250-268 (2007).

${ }^{11}$ Y. Xu, Z. C. Zheng, and D. K. Wilson, "Simulation of turbulent wind noise reduction by porous windscreens using high-order schemes," J. Comput. Acoust. 18, 321-334 (2010). 
${ }^{12}$ Y. Xu, Z. C. Zheng, and D. K. Wilson, "A computational study of the effect of windscreen shape and flow resistivity on turbulent wind noise reduction," J. Acoust. Soc. Am. 129, 1740-1747 (2011).

${ }^{13}$ C. Zwikker and C. W. Kosten, Sound Absorbing Materials (Elsevier, New York, 1949), pp. 18-24.

${ }^{14}$ J. B. Keller, "Diffraction by an aperture," J. Appl. Phys. 28, 426-444 (1957).

${ }^{15}$ J. B. Keller, "Geometrical theory diffraction," J. Opt. Soc. Am. 52, 116-130 (1962).

${ }^{16}$ A. D. Pierce, "Diffraction of sound around corners and over wide barriers," J. Acoust. Soc. Am. 55, 941-955 (1974).

${ }^{17}$ T. Kawai, "Sound diffraction by a many-sided barrier or pillar," J. Sound Vib. 79, 229-242 (1981).

${ }^{18}$ W. J. Hadden and A. D. Pierce, "Sound diffraction around screens and wedges for arbitrary point source location," J. Acoust. Soc. Am. 69, 1266-1276 (1981).

${ }^{19}$ H. S. Kim, J. S. Kim, H. J. Kang, B. K. Kim, and S. R. Kim, "Sound diffraction by multiple wedges and thin screens," Appl. Acoust. 66, 1102-1119 (2005).

${ }^{20}$ D. Chu, T. K. Station, and A. D. Pierce, "Higher-order acoustic diffraction by edges of finite thickness," J. Acoust. Soc. Am. 122, 3177-3193 (2007).
${ }^{21} \mathrm{H}$. Min and X. Qiu, "Multiple acoustic diffraction around rigid parallel wide barriers," J. Acoust. Soc. Am. 126, 179-186 (2009).

${ }^{22}$ E. Premat and Y. Gabillet, "A new boundary-element method for predicting outdoor sound propagation and application to the case of a sound barrier in the presence of downward refraction," J. Acoust. Soc. Am. 108, 2775-2783 (2000).

${ }^{23}$ T. Ishizuka and K. Fujiwara, "Performance of noise barriers with various edge shapes and acoustical conditions," Appl. Acoust. 65, 125-141 (2004).

${ }^{24}$ J. P. Berenger, "A perfectly matched layer for the absorption of electromagnetic waves," J. Comput. Phys. 114, 185-200 (1994).

${ }^{25} \mathrm{~F}$. Q. Hu, "On absorbing boundary conditions for linearized Euler equation by a perfectly matched layer," J. Comput. Phys. 129, 201-219 (1996).

${ }^{26} \mathrm{~F}$. Q. Hu, "A perfectly matched layer absorbing boundary condition for linearized Euler equations with a non-uniform mean-flow," J. Comput. Phys. 208, 469-492 (2005).

${ }^{27}$ D. Heimann, "On the efficiency of noise barriers near sloped terrain-a numerical study," Acta Acust. Acust. 96, 1003-1011 (2010).

${ }^{28} \mathrm{P}$. M. Morse and K. U. Ingard, Theoretical Acoustics (McGrawHill, New York, 1968), pp. 252-255. 